\title{
Evaluación de la resistencia al choque térmico de recubrimientos de estructura gradual obtenidos mediante proyección plasma
}

\author{
J.M. GUILEMANY, S. ARMADA, F. CLIMENT \\ Centro de Proyección Térmica (CPT). Ingeniería de Materiales. Dept. Enginyeria Química i Metal·lúrgia. \\ Universitat de Barcelona. 08028 Barcelona
}

\begin{abstract}
La creciente demanda de mayores temperaturas de trabajo y potencia en el sector de la aviación promovió el desarrollo de las barreras térmicas (TBCs) obtenidas por la técnica de proyección térmica plasma (APS). Éstas se componen de una capa de anclaje y una capa cerámica aislante. El espectacular aumento en el uso de las TBCs hace que se requieran recubrimientos cada vez más específicos en función de cada aplicación. Para mejorar la vida de los recubrimientos frente a los choques térmicos se han desarrollado los recubrimientos graduales. Éstos constan de una capa con variación gradual de los componentes, eliminando las intercaras entre partes con diferentes coeficientes de expansión térmica y minimizando las tensiones que se producen durante los cambios bruscos de temperatura.

En el presente trabajo se han optimizado las condiciones de proyección para la obtención de capas graduales y se ha comparado su resistencia al choque térmico con recubrimientos bicapa convencionales. Las condiciones de proyección se determinan de forma que sea posible la proyección conjunta de dos materiales con temperaturas de fusión muy diferentes, como son el NiCr y la $\mathrm{ZrO} 2$. De esta forma es posible obtener recubrimientos de estructura gradual utilizando únicamente una pistola. La resistencia al choque térmico de los recubrimientos graduales ha sido comparada con recubrimientos bicapa convencionales, obteniéndose una mejora de la vida media al eliminarse de forma gradual las superficies de contacto, a diferencia de los recubrimientos bicapa, donde se encuentran en contacto dos recubrimientos con Coeficientes de Expansión Térmica muy diferentes
\end{abstract}

Palabras clave: Proyección Térmica, Barreras térmicas, Choque térmico

Thermal shock resistance of functionally graded coatings obtained by plasma thermal spraying

Power increasing demand in aeronautical engines promoted the development of thermal barrier coatings (TBCs) obtained by Atmospheric Plasma Spray (APS). The spectacular growth in their applications leads to the demand of a very specific TBC. One of the specific roles that must achieve some TBCs is to withstand severe thermal shocks. Functionally Graded Coatings (FGC) can improve their lives due to the lack of interfaces between two layers with very different thermal expansion coefficient (TEC) (for example a metallic layer and a ceramic layer). Differences in TEC increase the internal stresses during the cooling of the parts.

In this work thermal spray conditions have been improved in order to obtain Functionally Graded Coatings. Thermal Shock Resistance of these coatings has been compared with conventional's one. Thermal Spray conditions have been used in order to spray two different powders with very different melting point. In that way it is possible to build up Functionally Graded Coatings with a unique spray gun. Thermal Shock Resistance of Coatings is improved due to the lack of interfaces between layers with different Thermal Expansion Coefficients unlike the conventional coatings where two layers of different materials are put together

Keywords: Thermal spray, Thermal barrier coatings, Thermal Shock resistance

\section{INTRODUCCIÓN}

La proyección térmica permite obtener recubrimientos de considerable grosor (desde $100 \mu \mathrm{m}$ hasta milímetros) en piezas que se han de someter a condiciones de trabajo extremas. En esta técnica se transmite energía térmica y cinética a un material en forma de hilo, varilla o polvo. El material es fundido y llega en estado pastoso o semipastoso a un substrato previamente preparado. La solidificación del material produce una unión mecánica con el substrato (1). Las barreras térmicas son un claro ejemplo de recubrimiento obtenido mediante la técnica de proyección térmica. Las barreras térmicas son ampliamente utilizadas en la actualidad para mejorar eficiencias en componentes que han de trabajar a elevadas temperaturas: álabes de turbina, pistones, válvulas, etc. La utilización de recubrimientos que disminuyan la temperatura del substrato permite aumentar la temperatura de trabajo (mejora en el rendimiento), aumentar la vida media de los componentes (disminución de costes), la disminución de refrigeración, etc.(2)

Las barreras térmicas suelen estar compuestas por una capa de anclaje que mejora la adherencia de los recubrimientos y evita la oxidación del substrato a las elevadas temperaturas de trabajo y de una capa cerámica con un bajo coeficiente de conductividad térmica que minimiza la transmisión de calor. El rápido crecimiento experimentado en los últimos años por este tipo de recubrimientos demanda la creación de 
recubrimientos cada vez más específicos: piezas sometidas a cambios bruscos de temperatura (choques térmicos) que provocan el rápido deterioro de las barreras térmicas convencionales debido a las diferencias de coeficiente de expansión térmica entre las diferentes partes que la componen. Para mejorar la vida de estos recubrimientos se desarrollaron las capas graduales, éstas constan de una zona donde la composición del recubrimiento varía gradualmente de forma que se eliminan las intercaras entre materiales que presentan coeficientes de expansión térmica diferentes.

La atmósfera en la que trabajan los recubrimientos tiene una gran importancia ya que las atmósferas oxidantes provocan la degradación prematura de los recubrimientos debido a la formación de una capa de óxido en la intercara metal-cerámico, que se ha determinado como factor crítico en la vida de las TBCs. Del mismo modo se espera un comportamiento similar en las capas graduales sometidas a ensayos de choque térmico en atmósfera oxidante y en atmósfera inerte.

\section{MÉTODO EXPERIMENTAL}

Los polvos seleccionados para la obtención de los recubri-

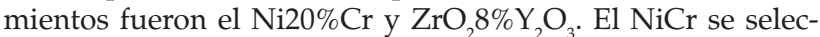
ciona por su resistencia a la oxidación a altas temperaturas, mientras que la $\mathrm{ZrO}_{2}$ es seleccionada por su baja conductividad térmica y su elevado coeficiente de expansión térmica. Los tamaños de partículas de los diferentes polvos han de ser lo más similar posible para que, durante la proyección de las capas graduales, la distribución de los polvos en el interior de la llama sea lo más homogénea y regular posible, siendo éstas $-53+16 \mu \mathrm{m}$ para el $\mathrm{NiCr}$ y $-45+11 \mu \mathrm{m}$ para la $\mathrm{ZrO}_{2}$. El substrato utilizado fue un acero $34 \mathrm{CrMo} 4$.

La proyección de las capas graduales conlleva la proyección de dos polvos de diferentes temperaturas de proyección en las mismas condiciones, lo que puede provocar un excesivo calentamiento de las partículas metálicas o un deficiente calentamiento de las partículas cerámicas al poseer éstas una temperatura de fusión mayor. Para la determinación de las condiciones energéticas óptimas se realizaron pruebas de proyección de los diferentes polvos independientemente (3). Las condiciones mostradas en la tabla I fueron seleccionadas para la obtención de las capas convencionales (bicapas) y capas graduales. Las capas graduales y bicapas se proyectaron en las mismas condiciones para obtener recubrimientos comparables, de otra forma las capas de $\mathrm{NiCr}$ y de $\mathrm{ZrO}_{2}$ de los dos recubrimientos serían completamente diferentes y por tanto tendrías diferente comportamiento frente a los choques térmicos.

TABLA I: CONDICIONES DE PROYECCIÓN UTILIZADAS PARA OBTENER LOS RECUBRIMIENTOS.

\begin{tabular}{|c|c|c|c|c|}
\hline $\operatorname{Ar}($ slpm) & $\mathrm{H}_{2}$ (slpm) & $\begin{array}{c}\text { Distancia de } \\
\text { proyección } \\
(\mathrm{mm})\end{array}$ & $\begin{array}{c}\text { Corriente } \\
(\mathrm{A})\end{array}$ & $\begin{array}{c}\text { Angulo de } \\
\text { inyección } \\
\left({ }^{\circ}\right)\end{array}$ \\
\hline 40 & 12 & 120 & 535 & 90 \\
\hline
\end{tabular}

Estas condiciones fueron utilizadas en la obtención de recubrimientos convencionales (capa de anclaje de $\mathrm{NiCr}$ y capa cerámica de $\mathrm{ZrO}_{2}$ ) (figura 1) y graduales (figura 2). Para su obtención se utilizó un equipo Plasma-Technik A-3000S con una pistola de plasma F4.

Las pruebas de choque térmico consistieron en someter a las piezas a ciclos de 10 minutos a $1000^{\circ} \mathrm{C}$ y enfriamiento en agua. Los choques térmicos se llevan a cabo en un horno con

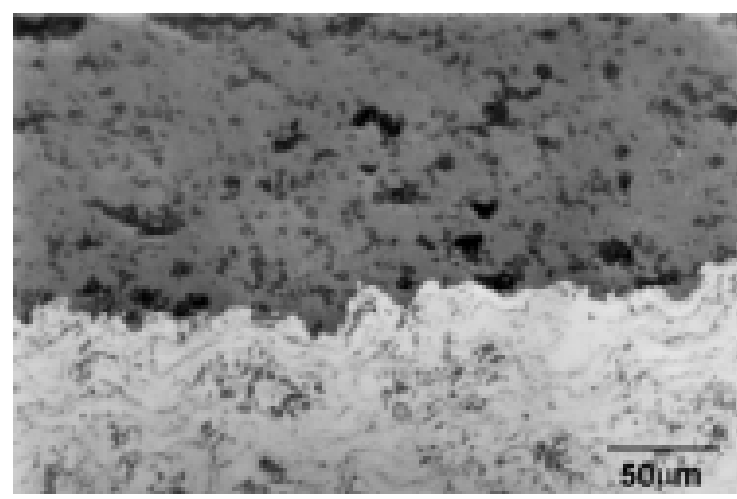

Figura 1: Bicapa convencional: intercara metal-cerámico

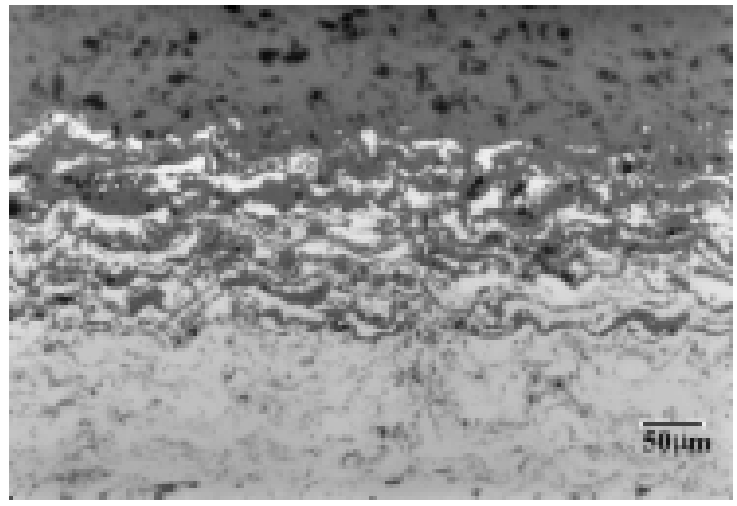

Figura 2: Capa gradual.

atmósfera oxidante y con atmósfera inerte (obtenida mediante un flujo continuo de Ar). Las piezas se consideran que han fallado cuando más del $10 \%$ de la capa cerámica ha sido eliminada por el efecto de los choques térmicos o se han formado grietas generalizadas a lo largo de toda la superficie

Las piezas, una vez han fallado, se evalúan mediante microscopía electrónica de scanning (JEOL JSM-5310 trabajando a $20 \mathrm{kV}$ ) y microanálisis EDS, tanto en sección transversal como en superficie libre.

\section{3.-RESULTADOS Y DISCUSIÓN}

Las capas graduales y bicapas obtenidas se basan en los sistemas $\mathrm{NiCr}+\mathrm{ZrO}_{2}$. Se utilizo $\mathrm{NiCr}$ como capa de anclaje en lugar del NiCrAlY o NiAl ya que la protección frente a la oxidación que este tipo de recubrimiento ofrece al acero es mejor que la ofrecida por los recubrimientos de NiCrAlY o NiAl (4). La optimización de las condiciones de proyección se realiza en función de la observación metalográfica de los recubrimientos metálicos y cerámicos proyectados de forma individual. Las condiciones de proyección con las que se obtienen los recubrimientos con menor índice de porosidad y mejor adherencia son las utilizadas para la obtención de la capa gradual y la bicapa. Las propiedades tecnológicas más relevantes de las condiciones tanto optimizadas como no optimizadas se encuentran recogidas en la bibliografía (5). Una vez determinadas las mejores condiciones se proyectan las capas graduales introduciendo en la llama los dos diferentes polvos y se determina su resistencia al choque térmico tanto en atmósfera oxidante como en atmósfera inerte. 
Los ensayos realizados muestran una mejora en la vida de los recubrimientos cuando éstos están compuestos por una capa gradual y al realizar el ensayo en atmósfera inerte. La vida de los diferentes recubrimientos se muestra en la tabla II. Se comprueba que los recubrimientos con estructura gradual llegan a mejorar la vida frente a los choques térmicos en atmósfera oxidante hasta en un $150 \%$, mientras que en atmósfera inerte la mejora es sólo de un $40 \%$. La vida de los recubrimientos en atmósfera inerte se mejora en todos los casos debido a la ausencia de oxidación del substrato, minimizándose por tanto las tensiones internas. De esta forma la rotura se produce básicamente por las tensiones creadas por las diferencias de coeficiente de expansión térmica de los diferentes componentes.

TABLA II: RESISTENCIA A LOS CHOQUES TÉRMICOS DE LOS DIFERENTES SISTEMAS

\begin{tabular}{|c|c|c|}
\hline Recubrimiento & Atmósfera & $\mathrm{N}^{\circ}$ de ciclos \\
\hline Bicapa & Oxidante & 10 \\
\hline Gradual & Oxidante & 25 \\
\hline Bicapa & Inerte & 25 \\
\hline Gradual & Inerte & 35 \\
\hline
\end{tabular}

La superficie libre de estos recubrimientos muestra un gran número de grietas en la superficie debido a las tensiones provocadas por los bruscos cambios de temperatura a los que se han sometido. La diferencia entre los coeficientes de expansión térmica de los diferentes materiales inducen tensiones en la capa cerámica provocando finalmente la rotura de ésta. Se observa la presencia de óxido de hierro en la superficie de las muestras ensayadas en atmósfera oxidante (figura 3) mientras que en las muestras ensayadas en atmósfera inerte no se observan restos de óxido de hierro (figura 4). Este hecho se explica por la formación de grietas longitudinales que alcanzan el substrato por donde el oxígeno puede alcanzar el acero provocando su oxidación, este proceso no ocurre en las pruebas realizadas en atmósfera inerte debido a la ausencia de oxígeno.

El estudio de la superficie libre de las muestras donde el recubrimiento de $\mathrm{ZrO}_{2}$ se ha eliminado muestra la ausencia de $\mathrm{NiCr}$ en su interior, indicando por tanto que la rotura se produce en el interior de la capa cerámica (figura 5). Los análisis realizados mediante EDS muestran únicamente la presencia de $\mathrm{Zr}$ y $\mathrm{O}$, con contenidos de $\mathrm{Ni}$ y $\mathrm{Cr}$ residual. Este tipo de rotura es típico de barreras térmicas que han sido sometidas a ensayos de choque térmico. Las grietas verticales que se producen por los cambios de temperatura se propagan en dirección horizontal a través de los poros y grietas entre splats (partículas que constituyen el recubrimiento) formados durante la proyección (6). La diferencia entre los coeficientes de expansión térmica del $\mathrm{NiCr}$ y de la $\mathrm{ZrO}_{2}$ induce tensiones durante los choques térmicos en el interior del recubrimiento cerámico que facilitan la propagación de las grietas a través de los poros debido a la relativa alta fragilidad del recubrimiento cerámico ya que datos bibliográficos señalan que tiene una tenacidad de $2.9 \mathrm{MPa} \cdot \mathrm{m}^{0.5}$ (7).

El estudio de la sección transversal de las piezas ensayadas muestra la presencia de grietas a lo largo del recubrimiento hasta alcanzar el substrato. Las muestras que se ensayaron en atmósfera oxidante presentan óxido en el interior de toda la grieta. En el final de la grieta se produce oxidación localizada, aunque ésta se extiende a lo largo de toda la intercara, reduciendo la adherencia del recubrimiento (figura 6).

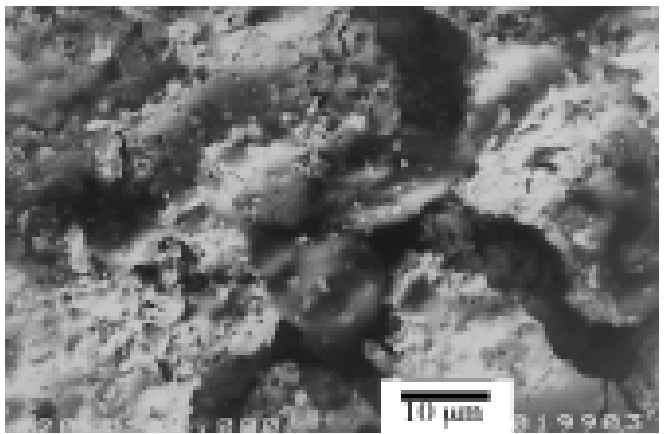

Figura 3: Grietas oxidadas en la superficie de muestras ensayadas en atmósfera oxidante

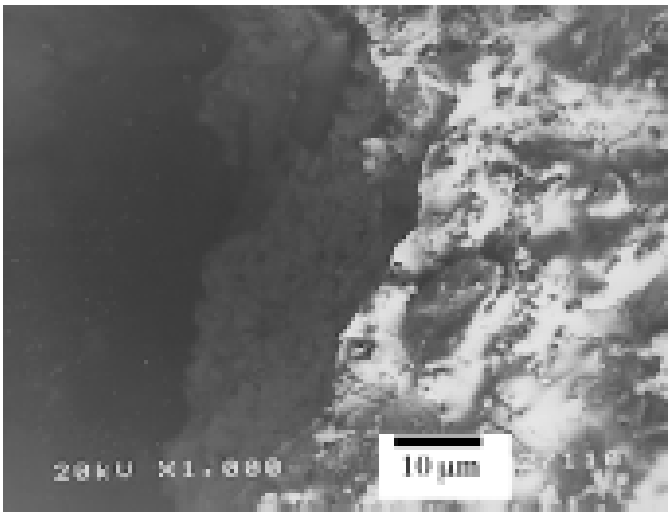

Fiura 4: Grieta en la superficie de muestras ensayadas en atmósfera inerte con ausencia de óxidos

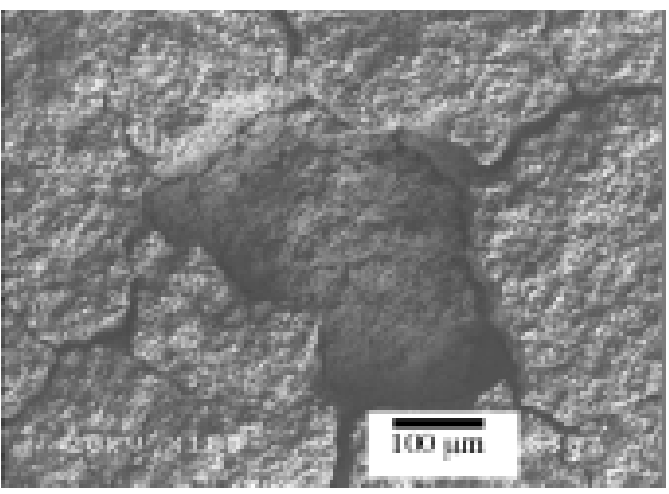

Figura 5: Deterioro del recubrimiento cerámico.

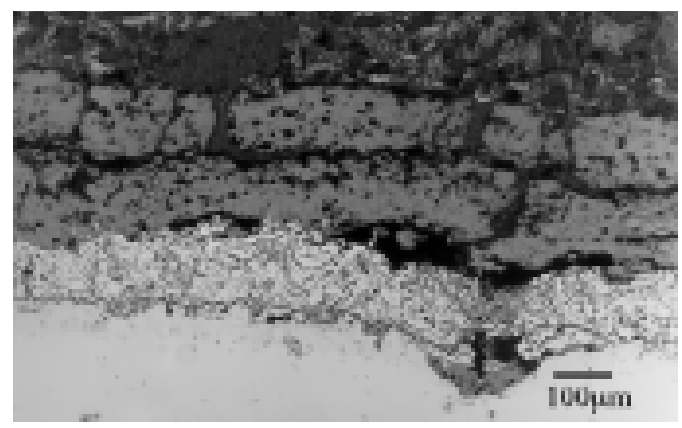

Figura 6: Sección transversal de grieta 
Las muestras ensayadas en atmósfera inerte muestran una pequeña zona oxidada que se produce durante la exposición de las muestras a la atmósfera durante el proceso de temple. La oxidación del substrato disminuye la vida de los recubrimientos al introducir tensiones por la formación de óxido en el interior de las grietas. Igualmente, el crecimiento del óxido de hierro en el interior de las grietas produce un aumento de las tensiones residuales en el recubrimiento cerámico, acelerando por tanto el proceso de rotura.

\section{CONCLUSIONES}

1. La vida a los efectos de choques térmicos de los recubrimientos con estructura gradual es significativamente mayor (superior a un 200\%) a la de los recubrimientos de estructura convencional. La minoración de las diferencias de coeficientes de expansión térmicos entre capas es el responsable de dicha mejora.

2. Los recubrimientos ensayados en atmósfera oxidante poseen una resistencia al choque térmico menor que los ensayados en atmósfera inerte que se cifra en un 30\% y $60 \%$ inferior para el recubrimiento con estructura gradual y bicapa, respectivamente. La formación de óxido de hierro al utilizarse la atmósfera oxidante, procedente de la oxidación del substrato, es la causante de dicho fenómeno ya que su crecimiento en las grietas aumenta el nivel de tensiones en el interior del recubrimiento, por lo que su resistencia a los choques térmicos disminuye.

3. La rotura de los recubrimientos tras el choque térmico se produce en el interior de la capa cerámica a través de la porosidad y grietas entre las partículas que constituyen el recubrimiento.

\section{AGRADECIMIENTOS}

Por el soporte del Ministerio de Ciencia y Tecnología en la concesión del proyecto C001999AX048 y de la Generalitat de Catalunya por el proyecto 1999SGR 00051, así como la concesión de una beca CIRIT 2000TDOC 00047

\section{BIBLIOGRAFÍA}

1. L.Pawlowski. "Coating build-up" pp. 108-163 en The Science and Engineering of Thermal Spray Coatings. John Wiley\&Sons, West Sussex (Inglaterra), 1995.

2. R.L.Jones. "Thermal Barrier Coatings" pp. 236-260 en Metallurgical and Ceramic Protective Coatings. Ed. K.H.Stern. Chapman\&Hall, Londres (Inglaterra), 1996

3. J.M. de Paco, S.Armada, J.M.Guilemany. "Improvement of Functionally Graded Coatings Obtained by Atmospheric Plasma Spraying" pp. 150-154 en EUROMAT'99. Dusseldorf. 1999. Wiley-VCH, Weinheim (Alemania), 2000.

4. J.M. Guilemany, J.M.de Paco, S.Armada. "Characterisation of bond coatings tested in oxidising environments" pp. 645-650 en UTSC99. Dusseldorf 1999. ASM International, 1998

5. S.Armada, Tesis doctoral, Universitat de Barcelona, Marzo 2001.

6. H.Ahn, S.H.Hong. "Design and Experiments of Graded Thermal Barrier Coatings by Plasma Sprayings" pp. 1635-1640 en Thermal Spray. Meeting the challenges of the $21^{\text {st }}$ century. Niza (Francia), 1998. ASM International, Ohio (Estados Unidos), 1998.

7. L.Pawlowski, The science and enginering of Thermal spray coatings, John Wiley\&Sons, Inglaterra, 1995.

Recibido: 16.11 .00

Aceptado: 10.11 .01 